\title{
A Comparison of Various Classification Techniques in Big Data Mining
}

\author{
Sunu fathima $\mathbf{T} \mathbf{H}^{\# 1}$, Binsu C Kovoor ${ }^{\# 2}$ Jaseena $\mathrm{K} \mathrm{U}^{\# 3}$ \\ \#1 M Tech Student, Division of Information Technology, Cochin University of Science \& \\ Technology, Kochi, Kerala, India \\ \#2 Assistant Professor, Division of Information Technology, Cochin University of Science \& \\ Technology, Kochi, Kerala, India \\ \#3 Research Scholar, Division of Information Technology, Cochin University of Science \& \\ Technology, Kochi, Kerala, India
}

\section{ABSTRACT}

Big Data is a term that refers to an extremely large volume of datasets such that traditional database and software techniques are inadequate to deal with them. The classification of big data becomes an important task in a wide variety of fields such as biomedicine, social media, marketing, etc. The recent advances in data gathering techniques have resulted in exponential growth of data. The characteristics of big data such as volume, diversity, and complexity make the analysis and knowledge extraction processes more complex. Thus standard data mining models need to be restructured to deal with this data. The main objective of this paper is to get deeper in big data classification and to compare various algorithms for the classification of big data including fuzzy and non-fuzzy algorithms. This paper also reviews some classification methods suitable for big data such as probabilistic classification, decision trees, rule-based methods, instance-based techniques, SVM methods, and neural networks.

Key words: Big data, classification, SVM, decision trees, fuzzy sets, neural networks, Naïve Bayes Classifier phylogeny.

\section{Corresponding Author: Binsu C Kovoor}

\section{INTRODUCTION}

Digital data is growing exponentially. The size of data and speed at which data is generating is always a criterion for business solutions. Collection of datasets that are so large and complex can be termed as Big Data. Big Data cannot process using traditional databases or software techniques. So we require some special tools for processing and managing this large amount of data like Hadoop, Apache Spark etc. It makes data storage and processing easier providing a helping hand in better decision making. Big Data can be described using 5 V's. Volume refers to a large amount of data is generating every second. Variety refers to different types of data like structured, unstructured, and semi-structured. Velocity is the speed at which data is generating and traveling. Veracity is the trustworthiness of the data. Value is the worth of data being 
DOI: https://dx.doi.org/10.26808/rs.ca.i8v2.07 International Journal of Computer Application (2250-1797)

Issue 8 Volume 2, March-April 2018

extracted. This five characteristics of big data make the process of big data analysis complicated. Therefore, we need to find efficient and effective techniques for big data classification.

Constructing fast and accurate classifiers for large data sets is an important task in data mining and knowledge discovery. Classification is one of the most important tasks in data mining. It is basically a machine learning attempt to predict the class of a new observation based on a training dataset which already contains a significant amount of classified data.

Basically, there are two types of classification techniques known as Supervised and Unsupervised techniques. In supervised classification techniques, training data contains labels indicating the class of the particular observation. Based on this training dataset, new data can be classified to find its class. Supervised techniques will usually have two steps called as Training stage and Testing stage. Training stage will analyze the training set and produce a classification model which will be in the form of some rules or algorithms. During testing, unseen data is given to the classification model to predict the class label of respective testing data. On the other hand, Unsupervised techniques do not have a training dataset. Unsupervised classification techniques are used to find clusters or groups of data by analyzing the relationship between given data.

The rest of the paper is organized into different sections. The section Classification Techniques introduce the concept of various supervised classification techniques and Section Related Works present an overview of related works. The section Comparative Study describes the comparison, results and analysis and conclusions are summarized in section Conclusion.

\section{CLASSIFICATION TECHNIQUES}

This section helps to familiarize with common supervised techniques used for Big Data classification such as Decision Trees, Support Vector Machine (SVM), Naïve Bayes Classification, K Nearest Neighbor (KNN) method, Fuzzy Techniques, Neural networks.

Decision Trees [1] are the most popular and simplest supervised method used for classification of large datasets. Easy interpretation, simple implementation, and easiness in understanding makes it one of the data analyst's favourite. Decision tree is a machine learning tool that represents a classification algorithm in the form of an acyclic graph. They classify data based on their feature values. A top-down greedy approach is used to recursively construct decision trees from training data. Decision Tree construction will start with the root node. Each internal node will represent a test on an attribute and each branch is the outcome of that test. Leaf nodes are the final outcome which is the class label. Each internal node with partitioned datasets will be expanded iteratively according to some splitting criteria. Quality measures such as Information Gain, Entropy, Gini Index etc. can be used to choose appropriate splitting criteria. ID3 by Quinlan [2], CART by Breiman et.al [3] are extended further to produce a large variety of wellknown decision tree classification algorithms.

Support Vector Machine (SVM) [4] is another popular classifier which can be defined formally by a separating hyperplane. The input to the algorithm is a labeled training set and the algorithm provides an optimal hyperplane that can categorize new examples. The focus of SVM algorithm is to find a hyperplane that provides the highest minimum distance towards the training data examples. Twice this distance is the margin which is an important parameter for every SVM 
DOI: https://dx.doi.org/10.26808/rs.ca.i8v2.07 International Journal of Computer Application (2250-1797)

Issue 8 Volume 2, March-April 2018

machine learning algorithm. A hyperplane which has the maximum margin of training data is known as an optimal hyperplane. The closest training examples to the hyperplane are generally known as support vectors. There are different notations used to define hyperplane formally. The training examples are arranged in a way such that the hyperplane will divide it from others by applying particular kernel equations. The kernel equations can be linear, Gaussian, Quadratic or any other forms [5]. It is the function of the kernel equation to convert the linearly non-separable data from one domain into another appropriate domain so that it will become linearly separable instances. Optimal hyperplane will decide the target variable value of the upcoming prediction.

Naïve Bayesian Classification [6] is a supervised technique which is based on Bayes theorem of probability. It works with an assumption of independence among predictors to predict the class of an unknown dataset. More specifically, it always assumes that the presence of a feature in a class is not related to the presence of another feature in the same class. This type of classifiers can be used to classify large datasets and it is easy to build. Naïve Bayes classifier can be used in multi class prediction especially in text classification. The assumption of independent predictors is a limitation to Naïve Bayes Classifier when coming to real life applications as there is little chance to get a set of completely independent predictors.

K Nearest Neighbor Algorithm (KNN) [7] is one of the simplest classification methods which deals with complex and arbitrary decision boundaries. Even though the learning is simple, but it is time-consuming compared to other classification methods. To classify an unlabelled test instance d, the KNN classifier will define K-neighborhood P as K-nearest neighbors of P. Count the number; $\mathrm{N}$ of training instances in $\mathrm{P}$ that belongs to a particular class $\mathrm{C}$. The new testing instance will be assigned a class label that has maximum instances in $\mathrm{P}$ neighborhood.

Fuzzy KNN Classifier [8] works based on the training set and fuzzy information generated from it. In this method, a class membership value is assigned to each sample. This class membership is defined as a function of the corresponding sample's distance from its respective $\mathrm{k}$ nearest neighbors of the training sample set. Each class in the training set will have a fraction of that sample defined using this membership value. A fuzzy KNN classifier gives some information on the certainty of the final decision taken on an unlabelled instance. Here it can assign the object to an unknown class instead of assigning it to a wrong class as most of the crisp systems doing.

Neural Networks [9] [10] are one of the most popular tools for classification. It belongs to the non-symbolic classification algorithm. There will be a learning stage which will generate maximum knowledge and it is then embedded as connection weights. The knowledge extracted during learning includes the correct class for each record and the weights associated. There is a disadvantage as it is black box [11]. This means that even though it gives correct output, it doesn't give a chance to know how it arrived at the correct result. This prevents it from using in real data mining problems.

\section{RELATED WORKS}

In this section, some classification methods proposed by different researchers for big data classification are presented.

Maillo et al. proposed a method to parallelize the KNN algorithm in [12]. It is based on MapReduce which is the most popular framework for handling big data. There will be a map and 
DOI: https://dx.doi.org/10.26808/rs.ca.i8v2.07 International Journal of Computer Application (2250-1797)

Issue 8 Volume 2, March-April 2018

reduce stage. The training data is split into different chunks of data and each chunk is fed into each map phase. Map phase will determine the $\mathrm{k}$ nearest neighbors available within a particular chunk of training data together with their distance values. It is then given to the reduce stage which determines the final $\mathrm{k}$ nearest neighbors for the whole training data by combining and sorting outputs of different map phases. Increasing the number of computing nodes can scale the $\mathrm{KNN}$ algorithm to process large datasets by parallelizing it.

Yang et al. [13] suggested a model that uses association rule mining to improve Naïve Bayes Classifier. Naïve Bayes Classifier is one of the most useful classification models which is based on independent assumptions of feature values. This principle becomes a drawback when dealing with real life scenarios. This can be overcome by combining Naïve Bayes Classifier with Association Rule Mining. Association Rule Mining detects the relation between different inputs. The above proposed model thus increases the accuracy of Naïve Bayes Classifier.

Bakry et al. [14] [15] described a MapReduce Fuzzy Techniques of Big Data Classification which implements a MapReduce paradigm using both fuzzy and crisp methods. The results obtained are compared with respect to different parameters. Four systems are implemented using MapReduce. The first proposed system is using the fuzzy KNN in the mapper and the mode in the reducer, the second proposed system is using the SVM in the mapper and the mode in the reducer, the third proposed system is using the SVM in the mapper and the soft labels in the reducer, and the fourth proposed system is using the SVM in the mapper and fuzzy Gaussian membership function in the reducer.

Singh et al. suggested a method known as DiP-SVM [16], a Distribution Preserving Kernel Support Vector Machine. For the proposed system, first order statistics and second order statistics of the entire data set are retained in each of the partitions which helps in obtaining local decision boundaries which agrees with global decision boundary. It partitions the data in a way that each subset is a sparse representation of the whole dataset. Sequential SVMs are trained on these reasonably sized subsets with many virtual machines.

Popescu and Keller proposed a system that can be used for the classification of highdimensionality datasets [17]. It combines two classification methods known as fuzzy k-nearest neighbor algorithm (FKNN) and feature reduction by random projection (RP). Here, values produced by class membership functions of FKNN and classification accuracy of each projection is fused to get better results.

Pei et al. proposed a new scheme that can construct an associative classifier for big probabilistic numerical data which works based on MapReduce framework [18]. The proposed system first illustrates the methods for original PND transformation following by fuzzy association rule mining. Redundant rules should be pruned from the final set of resultant association rules. An associative classifier is finally built on MapReduce paradigm.

Gamez et al. presented a system that uses an incremental approach to classify big data using fuzzy rules [19]. It implements an evolutionary fuzzy rule learning algorithm for classification known as IFRA using NSLV-AR kernel. This system provides a new rule model along with a mechanism to combine rules. Each new rule will be added to the database where all existing 
DOI: https://dx.doi.org/10.26808/rs.ca.i8v2.07

rules are stored. This rules will be analyzed then to prune redundant ones and combine similar rules to improve the efficiency of the classifier.

Na'el M. Abu-halaweh proposed FDT 1.0: An Improved Fuzzy Decision Tree Induction Tool [20], which is a software tool that will produce fuzzy decision trees to classify new unlabeled data instances. The proposed system is based on an improved version of the fuzzy ID3 (FID3) algorithm.

Erin-Elizabeth A. Durham et al. described a system known as "FDT 2.0: Improving scalability of the fuzzy decision tree induction tool - integrating database storage," [21] which is an improved version of FDT 1.0 [20]. It integrates FDT 1.0 tool with a database that will store all fuzzy rules at the time when they derived for the first time.

\section{COMPARATIVE STUDY}

In this section, the results of different classification methods discussed in the previous section III are analyzed and compared.

Parallelizing the K Nearest Neighbor Algorithm [12] can increase scalability by increasing the number of computing nodes. Thus KNN algorithm can deal with very large datasets. On the other hand, it will provide exact classification accuracy as the normal KNN algorithm. The limitation of Naïve Bayes Classifier can be overcome by combing it with Association Rule Mining [13]. This will increase the accuracy of the Naïve Bayes Classifier. Results of different fuzzy and crisp methods are compared using different datasets [14] [15]. Fuzzy methods outperform crisp methods in all aspects.

In DiP-SVM, only relevant local support vectors which are in agreement with the global separating hyperplane are produced [16]. It will reduce the loss of accuracy. It also reduces communication overhead since it transfers only relevant points to the next level. This leads to increase in speed of execution for larger datasets and also makes it suitable for implementing in cloud environments. Producing an ensemble classifier by combining an FKNN Classifier with Random projection [17] will increase the speed of classification and improve the accuracy as it can utilize the advantages of both classifiers. An associative classifier is built for big probabilistic numerical data in [18]. Therefore, it can process uncertain data with good accuracy. The use of MapReduce paradigm leads to faster classification.

Evolutionary fuzzy rule learning algorithm [19] is compared with an algorithm for learning fuzzy rule based on MapReduce paradigm. The results show that the proposed new incremental approach obtains a reduced and a compact number of rules. It also shows good results in comparison with the parallel algorithm. It gives similar results in terms of prediction capacity. The FDT 1.0 tool proposed in [20] is a scalable system that can deal with a large amount of datasets. It can deal with uncertain data since fuzzy set generation is combined with decision tree mechanism. FDT 2.0 [21] is an improved version of FDT 1.0 [20] which uses the advantage of integrating the former system with a database. So each time a new decision is needed, the system doesn't need to recalibrate from scratch. It is also scalable and can deal with uncertain data in a better way. The advantages and disadvantages of various classification methods discussed above are listed in Table 1. 
DOI: https://dx.doi.org/10.26808/rs.ca.i8v2.07 International Journal of Computer Application (2250-1797)

Issue 8 Volume 2, March-April 2018

Table 1: Comparison of Classification methods

\begin{tabular}{|c|c|c|c|c|}
\hline $\begin{array}{l}\text { Sl. } \\
\text { No. }\end{array}$ & Author & $\begin{array}{c}\text { Classification } \\
\text { Method }\end{array}$ & Advantages & Disadvantages \\
\hline 1 & $\begin{array}{l}\text { Maillo et al., } \\
2015 \text { in [12] }\end{array}$ & $\begin{array}{l}\text { MapReduce } \\
\text { based KNN } \\
\text { approach }\end{array}$ & $\begin{array}{l}>\text { Increase in scalability } \\
>\text { Deals with large } \\
\text { datasets }\end{array}$ & $\begin{array}{l}\text { Accuracy not so } \\
\text { better }\end{array}$ \\
\hline 2 & $\begin{array}{l}\text { Yang et al., } \\
2016 \text { in [13] }\end{array}$ & $\begin{array}{l}\text { Naïve Bayes } \\
\text { Classifier with } \\
\text { association } \\
\text { rule mining }\end{array}$ & $\begin{array}{l}>\text { Increased Accuracy } \\
>\text { Suitable for real data } \\
\text { applications }\end{array}$ & $\begin{array}{l}\text { Increased } \\
\text { complexity }\end{array}$ \\
\hline 3 & $\begin{array}{l}\text { Bakry et al., } \\
2016 \text { in [14] }\end{array}$ & $\begin{array}{c}\text { Fuzzy } \\
\text { technique }\end{array}$ & $\begin{array}{l}\text { Accuracy is more than } \\
\text { crisp techniques }\end{array}$ & $\begin{array}{l}\text { More } \\
\text { complexity }\end{array}$ \\
\hline 4 & $\begin{array}{l}\text { Singh et al., } \\
2017 \text { in [16] }\end{array}$ & DiP SVM & $\begin{aligned}> & \text { High accuracy } \\
> & \text { Low communication } \\
& \text { overhead } \\
> & \text { High execution speed } \\
> & \text { Possible to implement } \\
& \text { in a cloud environment }\end{aligned}$ & $\begin{array}{l}\text { Error in data } \\
\text { partitioning can } \\
\text { lead to } \\
\text { misclassificatio } \\
\mathrm{n}\end{array}$ \\
\hline 5 & $\begin{array}{l}\text { Popescu and } \\
\text { Keller, } 2016 \\
\text { in [17] }\end{array}$ & Fuzzy- KNN & $\begin{array}{l}>\text { Increase in speed of } \\
\text { classification } \\
>\text { Increase in accuracy }\end{array}$ & $\begin{array}{l}\text { Complex to } \\
\text { handle }\end{array}$ \\
\hline 6 & $\begin{array}{c}\text { Pei et al., } \\
2016 \text { in }[18]\end{array}$ & $\begin{array}{l}\text { MapReduce } \\
\text { based fuzzy } \\
\text { association } \\
\text { classifier }\end{array}$ & $\begin{array}{l}>\text { Classify uncertain data } \\
>\text { Good accuracy } \\
>\text { Faster classification }\end{array}$ & $\begin{array}{l}\text { Can process } \\
\text { only numerical } \\
\text { data }\end{array}$ \\
\hline 7 & $\begin{array}{l}\text { Gamez et al., } \\
2016 \text { in [19] }\end{array}$ & $\begin{array}{c}\text { An } \\
\text { Incremental } \\
\text { approach to } \\
\text { fuzzy } \\
\text { classification } \\
\text { rules }\end{array}$ & $\begin{array}{l}\text { Generate less number } \\
\text { of classification rules }\end{array}$ & $\begin{array}{l}\text { No } \\
\text { improvement in } \\
\text { prediction } \\
\text { capacity }\end{array}$ \\
\hline 8 & $\begin{array}{c}\text { Na'el M. } \\
\text { Abu-halaweh, } \\
2010 \text { in [20] }\end{array}$ & $\begin{array}{c}\text { FDT 1.0: } \\
\text { Fuzzy } \\
\text { Decision Tree }\end{array}$ & $\begin{array}{l}>\text { Scalable } \\
>\text { Classifies uncertain } \\
\text { data }\end{array}$ & $\begin{array}{l}>\text { Less accuracy } \\
>\text { Low execution } \\
\text { speed }\end{array}$ \\
\hline 9 & $\begin{array}{c}\text { Erin- } \\
\text { Elizabeth A. } \\
\text { Durham et } \\
\text { al., } 2014 \text { in } \\
\text { [21] }\end{array}$ & $\begin{array}{c}\text { FDT 2.0: } \\
\text { Fuzzy } \\
\text { Decision Tree }\end{array}$ & $\begin{array}{ll}> & \text { More scalable } \\
> & \text { No need to recalibrate } \\
& \text { from scratch }\end{array}$ & $\begin{array}{l}\text { Error in a single } \\
\text { rule can affect } \\
\text { the future } \\
\text { classification }\end{array}$ \\
\hline
\end{tabular}

\section{CONCLUSION}

Classification is the most important task in mining interesting patterns from big data. It refers to predicting the class label of a new instance based on a training dataset. In this paper, we conducted a detailed study on different supervised classification methods that can be applied for 
DOI: https://dx.doi.org/10.26808/rs.ca.i8v2.07 International Journal of Computer Application (2250-1797)

Issue 8 Volume 2, March-April 2018

big datasets and compared the results of those methods based on different parameters such as scalability, complexity, accuracy etc. New classification approaches are inventing day by day by overcoming the limitations of already existing algorithms and modifying them. The comparative study on this area will be helpful for researchers to decide which method to be used to handle their big datasets based on the characteristics of their data.

\section{REFERENCE}

[1] L. Rokach and O. Maimon, Data mining with decision trees: Theory and applications. World scientific, 2014.

[2] J. R. Quinlan, "Induction of decision trees," Machine learning, vol. 1, no. 1, pp. 81-106, 1986.

[3] L. Breiman, J. Friedman, C. J. Stone, and R. A. Olshen, Classification and regression trees. CRC press, 1984.

[4] K. Muller, S. Mika, G. Ratsch et al., "An introduction to kernel-based learning algorithms," IEEE Transactions On Neural Networks, vol. 12, no. 2, pp. 181-201, 2001.

[5] Imen Trabelsi and Dorra Ben Ayed, "On the use of different feature extraction methods for linear and non-linear kernels," 2012 6th International Conference on Sciences of Electronics, Technologies of Information and Telecommunications (SETIT), pp.797-802, 2012.

[6] Harry Zhang, Jiang Su, J.F Boulicaut, F. Esposito, F. Giannotti, D. Pedreschi, "Naïve Bayesian Classifiers for Ranking", Proceedings of the $15^{\text {th }}$ European Conference for Machine Leaning(ECML-04), pp. 501-512, 2004.

[7] B. V. Dasarathy, "Visiting nearest neighbor-A survey of nearest neighbor classification techniques," in Proceedings of International Conference for Cybern. Soc, pp. 630-636, 1977.

[8] James M. Keller, Michael R. Gray, and James A. Givens, JR, "A Fuzzy K-Nearest Neighbor Algorithm," IEEE Transactions On Systems, Man, And Cybernetics, Vol. SMC-15, No. 4, July/August 1985.

[9] G. Towell and W. Shavlik, "Refining symbolic knowledge using neural networks," in Machine Learning: A Multistrategy Approach, CA: Morgan Kaufmann, 1994, pp. 405429.

[10] S. C. Park, S. M. Lam, and A. Gupta, "Rule extraction from neural networks: Enhancing the explanation capability," J. Expert Syst., vol. 2, pp. 57-71, 1995.

[11] J. M. Benitez, J. L. Castro, and I. Requena, "Are neural networks black boxes?" IEEE Trans. Neural Networks, vol. 8, Dec. 1997.

[12] Jesus Maillo, Isaac Triguero, Francisco Herrera, "A MapReduce-based k-Nearest Neighbor Approach for Big Data Classification," 2015 IEEE Trustcom / Big Data SE/ISPA, vol.2, pp.167-172,2015.

[13] Tianda Yang, Kai Qian, Dan Chia-Tien Lo, Ying Xie, Yong Shi, Lixin Tao, "Improve the Prediction Accuracy of Naïve Bayes Classifier with Association Rule Mining,"2016 IEEE 2nd International Conference on Big Data Security on Cloud, IEEE International Conference on High Performance and Smart Computing, IEEE International Conference on Intelligent Data and Security, pp.129-133,2016.

[14] Malak El Bakry, Soha Safwat, Osman Hegazy, "A MapReduce Fuzzy Techniques of Big Data Classification," 2016 SAI Computing Conference (SAI), pp.118-128, 2016. 
DOI: https://dx.doi.org/10.26808/rs.ca.i8v2.07

[15] Soha Safwat Labib, "A Comparitive Study to Classify Big Data Using Fuzzy Techniques," 2016 5th International Conference on Electronic Devices, Systems and Applications (ICEDSA), pp.1-6, 2016.

[16] Dinesh Singh, Debaditya Roy, C. Krishna Mohan, "DiP-SVM: Distribution Preserving Kernel Support Vector Machine for Big Data," IEEE Transactions on Big Data, vol.3, pp.79-90, 2017.

[17] Mihail Popescu, James M. Keller, "Random projections fuzzy k-nearest Neighbor (RPFKNN) for big data classification,"2016 IEEE International Conference on Fuzzy Systems (FUZZ-IEEE), pp.1813-1817, 2016.

[18] Bin Pei, Fenmei Wang, Xiuzhen Wang, "Research on MapReduce-based fuzzy associative classifier for big probabilistic numerical data," 2016 IEEE International Conference on Internet of Things (iThings) and IEEE Green Computing and Communications (GreenCom) and IEEE Cyber, Physical and Social Computing (CPSCom) and IEEE Smart Data (SmartData), pp.903-906,2016.

[19] Juan Carlos David Garc'1a, Antonio Gonz'alez, Ra'ul P'erez, "On the use of an incremental approach to learn fuzzy classification rules for big data problems," 2016 IEEE International Conference on Fuzzy Systems (FUZZ-IEEE), pp.1413-1420,2016.

[20] Na'el M. Abu- halaweh, Robert W. Harrison, "FDT 1.0: An Improved Fuzzy Decision Tree Induction Tool," 2010 Annual Meeting of the North American Fuzzy Information Processing Society, pp.1-5,2010.

[21] Erin-Elizabeth A. Durham, Xiaxia Yu, Robert W. Harrison," FDT 2.0: Improving scalability of the fuzzy decision tree induction tool - integrating database storage," 2014 IEEE Symposium on Computational Intelligence in Health Care and e-health (CICARE), pp.187-190,2014. 